\title{
Factores que influyen sobre la actividad microbiana en basureros de hormigas cortadoras de hojas
}

\author{
Anahí Fernández ${ }^{1}$; Alejandro G. FarjI-Brener ${ }^{\varpi, 1,2} \&$ Patricia Satti $^{1,3}$ \\ 1. Centro Regional Universitario Bariloche, Universidad Nacional del Comahue Bariloche, Argentina. 2. Laboratorio Ecotono, \\ CRUB-UNComa, INIBIOMA-CONICET. 3. Universidad Nacional de Río Negro
}

\begin{abstract}
Resumen. Los microorganismos del suelo cumplen un papel relevante en el ciclado de nutrientes. En consecuencia, conocer los factores que influyen sobre su actividad es de fundamental importancia para comprender mejor el funcionamiento de los ecosistemas terrestres. Empleando a los basureros externos de la hormiga cortadora Acromyrmex lobicornis como sistema modelo, analizamos la asociación de la diversidad de compuestos carbonados y la concentración de carbono con la respiración potencial de microorganismos en ese sustrato. En muestras de basureros en condiciones controladas de laboratorio estimamos la concentración de carbono y la respiración de los microorganismos. En paralelo, cuantificamos la diversidad de la dieta de cada nido como un estimador de la diversidad de compuestos carbonados en sus basureros. La actividad de los microorganismos no se relacionó con la concentración de carbono en los basureros pero mostró una relación de tendencia positiva con la riqueza de la dieta de las hormigas. Como la diversidad de compuestos carbonados en el basurero podría reflejar parcialmente la diversidad de plantas cosechadas por las hormigas, este resultado sugiere que la actividad de los microorganismos descomponedores depende parcialmente de la diversidad de recursos para descomponer. Este trabajo ejemplifica cómo las hormigas cortadoras afectan al suelo en ambientes áridos en donde la materia orgánica es limitante; e ilustra de forma novedosa cómo la pérdida de la diversidad vegetal, al empobrecer la dieta de las hormigas, puede afectar indirecta y negativamente la actividad de los descomponedores.
\end{abstract}

[Palabras clave: Acromyrmex lobicornis, descomposición, diversidad, productividad, Patagonia]

\begin{abstract}
AвSTRACт. Factors influencing microbial activity in dumps leaf cutter ants: Soil microorganisms play a key role in the process of nutrient cycling. Therefore, the knowledge of the factors that influence its activity is vital for a better understanding of terrestrial ecosystem function. We use external refuse dumps from the leaf-cutting ant Acromyrmex lobicornis such as a model system to determine the influence of the concentration and diversity of organic matter on the activity of soil decomposers. We estimated the concentration of carbonate compounds and the respiration rate of microorganisms in samples of refuse dumps under lab conditions. We also estimated the diversity of the ant diet like a proxy of the diversity of organic compounds in their refuse dumps. The activity of microorganisms did not show association with the concentration of carbonate compounds, but showed a tendency to a positive association with the diversity of the ant diet. Since the diversity of organic matter in refuse dumps depends on the diversity of the plants harvested by ants, these results partially support the idea that the activity of soil microorganisms partially depends on the diversity of resources to decompose. This work exemplify how leaf-cutting ants affect soil properties in arid environments where the availability of organic matter is limiting, and illustrate a novel way how the reduction of vegetation diversity may indirectly affect the productivity of a system through the impoverish of the leaf-cutting ant's diet.
\end{abstract}

[Keywords: Acromyrmex lobicornis, decomposition, diversity, productivity, Patagonia]

\section{INTRODUCCIÓN}

La descomposición de la materia orgánica que realizan los microorganismos del suelo es indispensable para el funcionamiento de los ecosistemas terrestres. Cuando los nutrientes vuelven al suelo como materia orgánica muerta no se encuentran disponibles para la vegetación, que sólo puede absorberlos en estado inorgánico. Es allí cuando los microorganismos descomponen la materia orgánica muerta y producen la mineralización de los nutrientes, proceso por el cual quedan nuevamente disponibles para las plantas $\mathrm{y}$, de forma indirecta, para el resto de la cadena trófica. En consecuencia, el estudio

$\bowtie$ alefarji@yahoo.com de los factores que afectan la actividad de los descomponedores es vital para comprender el funcionamiento de los ecosistemas terrestres.

La actividad de los descomponedores normalmente depende factores abióticos como la temperatura y la humedad ambiental (Orchard \& Cook 1983; Thomey et al. 201; Caprez et al. 2012). Sin embargo, factores intrínsecos de la materia orgánica a descomponer, como, por ejemplo, la concentración de carbono y la diversidad de compuestos carbonados pueden también afectar la tasa de respiración microbiana. Por un lado, una mayor concentración de

Recibido: 27 de mayo de 2013; Fin de arbitraje: 2 de agosto; Última versión: 2 de diciembre; Aceptado: 10 de diciembre. 
carbono "per se" puede ofrecer una mayor disponibilidad de recursos para degradar (i.e., una mayor disponibilidad de alimento para los microorganismos), permitiendo un incremento en la actividad de los descomponedores si no hay limitaciones por otros factores. Por otra parte, una mayor diversidad de compuestos carbonados también puede influir sobre la actividad de los descomponedores si favorece la coexistencia de grupos especializados en la descomposición de estos diferentes compuestos, grupos que pueden interactuar entre ellos mediante procesos de facilitación o complementación (Naeem et al. 2000; Loreau 2001). En este último caso, el resultado esperado es que la actividad microbiana se incremente con los aumentos en la diversidad de la materia orgánica disponible (Gessner et al. 2010). Comprender la importancia relativa de estos factores puede ser vital para realizar de forma adecuada trabajos de conservación o restauración de ecosistemas degradados.

En ambientes áridos, la cobertura vegetal y la biomasa animal son relativamente bajas y poco diversas, lo cual restringe la disponibilidad y la variedad de materia orgánica en el suelo; esto limita la actividad de descomponedores (Orchard \& Cook 1983; Naeem et al. 2000). En estos ambientes, las hormigas son consideradas como un grupo clave en el proceso de incorporación de materia orgánica al suelo y ciclado de nutrientes (Bestelmeyer \& Wiens 2003). En particular, las hormigas cortadoras de hojas (Atta y Acromyrmex) son uno de los grupos cuyos efectos sobre el suelo son muy drásticos debido al gran tamaño de sus colonias, su larga vida y la alta densidad de nidos (Wirth et al. 2003). Este tipo de hormigas cortan material vegetal para cultivar un hongo dentro del nido (Leucoagaricus gogylophorus), con el cual alimentan a sus larvas. Una vez ingresados los fragmentos vegetales al hormiguero, los mismos son fraccionados hasta convertirse en una pasta. Esta pasta es incorporada por las hormigas a los jardines de hongo. Dado que estos hongos son incapaces de degradar por completo el tejido vegetal (Abril \& Bucher 2002, 2004; Moller 2011), este proceso de degradación por el hongo genera grandes cantidades de desechos orgánicos. Estos desechos se componen básicamente de restos de tejido vegetal no degradado (e.g., compuestos carbonados) y son desechados en basureros ubicados en cavidades especiales dentro del nido o sobre la superficie del suelo, dependiendo de la especie de hormiga cortadora (Farji-Brener \& Medina 2000). Estos basureros, de contextura fácilmente distinguible (Foto 1), son un importante aporte de materia orgánica al suelo, lo que favorece el ciclado de nutrientes y el establecimiento y desarrollo de la vegetación aledaña (FarjiBrener \& Illes 2000).

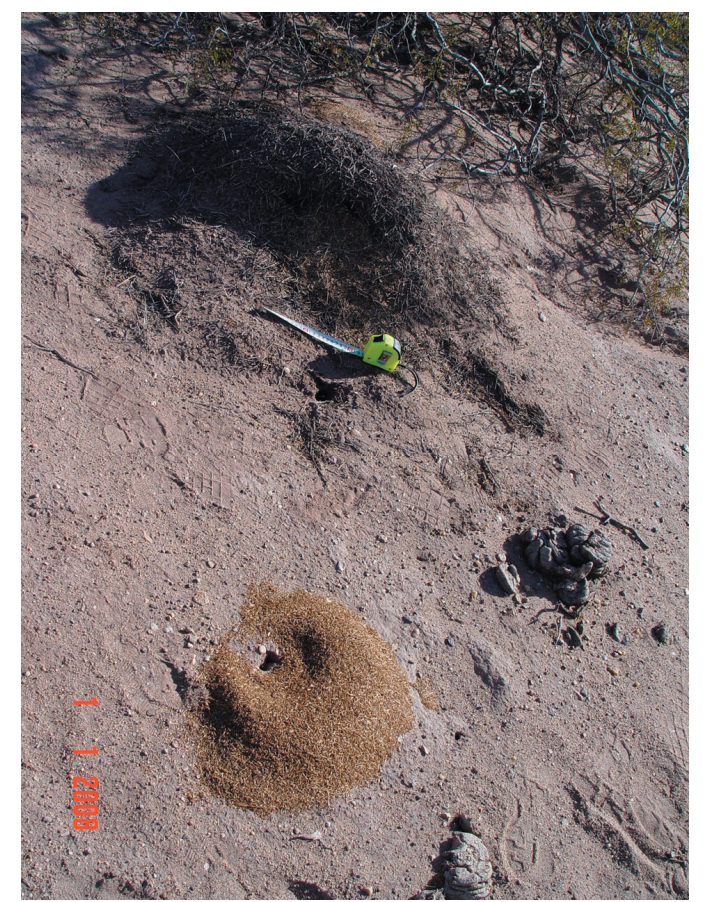

Foto 1. Nido de la hormiga cortadora Acromyrmex lobicornis. Se puede observar el domo externo del hormiguero formado por palitos y material vegetal seco (arriba) y su basurero externo en donde se depositan los restos vegetales no degradados por el hongo (abajo).

Photo 1. Nest of the leaf-cutting ant Acromyrmex lobicornis. Is easy to visualizes the external ant-mound building by dry sticks and soil (above), and the external refuse dump were the rest of plant material not processed by the fungus is deposited (below).

En las regiones áridas de la Patagonia, el establecimiento, crecimiento y reproducción de las plantas está limitado por la disponibilidad de agua y materia orgánica (Aguiar \& Sala 1994; Satti et al. 2003). Por lo tanto, los parches de fertilidad generados por las hormigas cortadoras tienen potencialmente una gran relevancia en estos ecosistemas. La única especie de hormiga cortadora de hojas en esta región es Acromyrmex lobicornis (Emery). Hay varias razonas por las cuales esta hormiga es un buen modelo para estudiar cómo características intrínsecas de la materia orgánica pueden influir sobre la actividad microbiana. Primero, porque la ubicación de sus basureros es en montículos externos bien definidos, lo cual facilita su estudio. Segundo, porque los basureros son un sustrato que naturalmente pueden variar en la diversidad 
de compuestos carbonados que lo componen. Como diferentes especies de plantas varían en su composición bioquímica, y al ingresar al nido este material es degradado por una misma especie de hongo en todas las colonias, es de suponer que cambios en la diversidad de dieta se expresen como cambios en la diversidad de compuestos orgánicos en la materia orgánica de los basureros (Abril \& Bucher 2004; Tadey \& Farji-Brener 2007; Scott et al. 2010; Moller et al. 2011). Finalmente, esta hormiga es un buen modelo porque se conoce que sus basureros albergan una importante cantidad de microorganismos descomponedores de materia orgánica (Farji-Brener 2010). Todas estas razones hacen de los basureros de $A$. lobicornis un buen sistema para analizar cómo ciertas características de la materia orgánica afectan la actividad de los descomponedores. En este trabajo, empleando a los basureros externos de la hormiga cortadora A. lobicornis, nosotros vamos a analizar si la actividad microbiana se relaciona con la diversidad de recursos carbonados (estimados a través de la diversidad de la dieta de las hormigas) y la concentración de carbono.

\section{Materiales y MéTOdos}

Los muestreos se desarrollaron en la estepa Patagónica y el Monte. La Estepa Patagónica es un ecosistema semiárido, tiene clima seco y frío con vientos fuertes del sector oeste. La temperatura media anual oscila entre 8 y $14{ }^{\circ} \mathrm{C}$ dependiendo de la exposición (sur y norte, respectivamente), mientras que la precipitación media anual es $400 \mathrm{~mm}$. Los suelos son de textura variable con predominancia de textura gruesa, pedregosos, ricos en carbonatos de calcio y pobres en materia orgánica (Del Valle 1998). La vegetación es principalmente arbustiva; con matorrales achaparrados, en cojín, con espinas, y si poseen hojas, son pequeñas. En menor proporción aparecen estepas herbáceas de pastos xerófilos o con adaptaciones a características edáficas particulares (Burkart et al. 1999). El muestreo en esta región fue realizado en áreas de estepa cercanas a Villa Llanquín y Dina Huapi, localidades ubicadas a 40 y $15 \mathrm{~km}$ de la ciudad de San Carlos de Bariloche, respectivamente. En estos sitios los arbustos más frecuentes son Discaria chacaye, D. articulata, Ochetophila trinervis, Berberis buxifolia, Mulinum spinosum y Senecio filaginoides y las hierbas Stipa speciosa, Festuca pallescens y Acaena splendens (Mermoz \& Martin 1986).

La región del Monte se caracteriza por un clima templado-árido y escasas precipitaciones. $\mathrm{La}$ temperatura media anual oscila entre 10 y $14{ }^{\circ} \mathrm{C}$ dependiendo de la exposición, y la precipitación media oscila entre 100 y $200 \mathrm{~mm}$. Los suelos son principalmente aridisoles, en su mayoría salinos y pedregosos (Burkart et al. 1999). La vegetación es escasa y predominan varias especies del género Larrea, Montea aphylla y Bougainvillea spinosa. También se encuentran Cassia aphylla, Prosopis torquata, Cercidium praecox, Chuquiraga erinacea y Prosopis alpataco (Cabrera 1976). En esta región, los muestreos fueron realizados alrededor, dentro de la ciudad de Neuquén, y a $80 \mathrm{~km}$ de la misma, en la costa del lago Mari Menuco.

\section{Especie de hormiga}

Acromyrmex lobicornis (Emery) es la especie de hormiga cortadora de hojas con el rango latitudinal más amplio de Argentina, abarcando desde los $23^{\circ}$ S hasta $44^{\circ} \mathrm{S}$ (Farji-Brener \& Ruggiero 1994). Es la única de este grupo que se encuentra en Patagonia, y es abundante en áreas disturbadas (Farji-Brener 2000). Como el resto de las hormigas cortadoras, utilizan el material vegetal cosechado para cultivar un hongo simbionte, principal alimento de las larvas. Esta especie recolecta especies de plantas mono y dicotiledóneas, y su dieta puede variar entre colonias dependiendo del ambiente en donde se encuentran (Pilati et al. 1997; Franzel \& Farii-Brener 2000). Los nidos pueden tener hasta 1 $m$ de profundidad, y sobre la superficie del suelo las hormigas construyen un domo con ramitas y suelo que puede alcanzar hasta $1 \mathrm{~m}$ de ancho y de alto. Dentro del montículo se encuentra el hongo que cultivan. Los desechos orgánicos producidos por el proceso de descomposición del material vegetal llevado a cabo por el hongo son depositados por las hormigas en un montículo sobre la superficie del suelo cercano al nido, en adelante "basurero" (Foto 1). Estos basureros tienen mayor capacidad de retención de agua (Farji-Brener \& Ghermandi 2004), mayor concentración de N, P, K y C que el suelo adyacente (Farji-Brener \& Tadey 2009) y mayor concentración de microorganismos que el suelo circundante (Farji-Brener 2010).

Recolección de muestras de basureros, respiración de microorganismos y estimación de la concentración de carbono

Para analizar la relación de la actividad de los descomponedores con la concentración de carbono y la diversidad de compuestos carbonados en los basureros, recolectamos basureros en 19 nidos adultos (domo $\sim 1 \mathrm{~m}$ de diámetro). Las muestras de basurero ( $\sim 300 \mathrm{~g}$ cada una) fueron sacadas de la capa superior del montículo para asegurar que los mismos correspondían a los últimos desechos que fueron depositados (o sea, correspondientes a la dieta estimada, ver explicación más adelante). Las muestras de basurero fueron llevadas al laboratorio y secadas al aire libre durante una semana para reducir a su mínima expresión la actividad microbiana. Luego se las conservó en recipientes cerrados durante dos meses hasta comenzar con la parte experimental.

Estimamos la actividad microbiana midiendo su respiración. Para ello las muestras fueron humedecidas con una cantidad de agua equivalente a $3 \mathrm{~mm}$ de precipitación $\left(10 \mathrm{~cm}^{3} \mathrm{H}_{2} \mathrm{O} / 190 \mathrm{~cm}^{3}\right.$ 
sustrato), valor que representa la lluvia promedio caída en una semana de primavera con baja precipitación (ver detalles del cálculo en Farji-Brener \& Ghermandi 2000, 2004). Luego de humedecer las muestras, éstas se dejaron estabilizar en bolsas de nylon cerradas (con una cámara de aire) durante diez días a una temperatura aproximada de $10^{\circ} \mathrm{C}$. La técnica utilizada para medir la respiración microbiana fue la de "trampas alcalinas" que mide la respiración a través de la evolución de C-CO (Anderson 1982; Cooperband \& Middleton 1996). Esta técnica se basa en cuantificar el $\mathrm{CO}_{2}$ liberado durante la oxidación de carbohidratos, a través de la reacción del mismo con $\mathrm{NaOH}$ (Kandeler 2007). Debido a la gran cantidad de materia orgánica de los basureros, la respiración se evaluó a las 72 h cómo se ha realizado para compost en trabajos anteriores (Iannotti et al. 1994; Tognetti et al. 2007; Leconte et al. 2009).

Para determinar la relación entre la respiración de los microorganismos y la concentración de carbono, determinamos la concentración de este elemento en las muestras de los basureros de los 19 nidos muestreados a través del método de combustión seca a alta temperatura, empleando un analizador de Carbono-Nitrógeno por combustión marca Thermofinnigan ${ }^{\circledR}$, modelo Flash 1112 . La metodología aplicada para procesar las muestras fue la normalmente utilizada en suelos, la que fue considerada como más adecuada por análisis anteriores realizados con este tipo de sustrato (ver detalles metodológicos en Nelson \& Sommer 1996) (Farji-Brener \& Ghermandi 2000, 2004; Tadey \& Farji-Brener 2007). El resultado fue expresado en $\mathrm{g} \mathrm{C} / \mathrm{kg}$ sustrato seco.

\section{Estimación de la dieta de A. lobicornis}

Como medida indirecta de la diversidad de compuestos carbonados en la basura se determinó la dieta en las 19 colonias de A. lobicornis muestreadas. Como el material vegetal cosechado por las hormigas es depositado en el basurero luego de ser procesado por el hongo, es lógico suponer que la diversidad vegetal de sus desechos va a depender de la diversidad de material vegetal que ingresa al nido. Dado que nuestro objetivo era determinar la existencia de una asociación entre la actividad de los descomponedores y la diversidad de compuestos orgánicos para degradar, elegimos hormigueros ubicados en sitios que presentaran variación en la diversidad y cobertura de plantas, esperando que esta variación en la oferta se reflejara en riqueza de la dieta de las hormigas. Para ello, elegimos hormigueros ubicados en ambientes con diferente nivel de disturbio (i.e., variable en la oferta de especies vegetales), que incluyeron desde plazas urbanas hasta parques nacionales (Apéndice S1, Información Suplementaria). Determinamos la composición de la dieta durante 2-3 días en cada nido durante la época del año y horas del día de máxima actividad forrajera, para abarcar la máxima diversidad de dieta posible. Esta época fue primavera-verano (años 2011 y 2012), y en horarios diurnos variables a la mañana y a la tarde (esta especie de hormiga presenta muy poca actividad en otoño y nula en invierno, así como en horarios con muy altas o muy bajas temperaturas). En los dos principales senderos de forrajeo de cada nido, durante 5 minutos en cada uno, colectamos los fragmentos acarreados por cada obrera que pasó por un punto fijo a 1-3 m de la entrada del nido. Los muestreos en cada sendero estaban espaciados por alrededor de 20 minutos y el número total de muestreos fue 10 por nido, los cuales fueron sumados para estimar la dieta de cada hormiguero. Los fragmentos cosechados fueron guardados en un sobre de papel y llevados al laboratorio para su posterior identificación taxonómica al menor nivel posible con la ayuda de un herbario y bibliografía (Haloua Gruneisen 1996). Para cada nido confeccionamos una lista de especies vegetales consumidas y su frecuencia relativa (Apéndice S2, Información Suplementaria). Con estos datos, determinamos la riqueza de la dieta (i. e., $\mathrm{S}=$ número de especies vegetales en la dieta) y su diversidad mediante el índice de ShannonWiener. Los palitos leñosos no fueron incluidos en la estimación de la diversidad de dieta porque estos ítems son empleados para el arreglo y expansión del domo del nido, y no son incorporados al jardín de hongos. El tiempo que transcurre entre el ingreso de los fragmentos vegetales al nido y su aparición en el basurero como desechos orgánicos es de 6 a10 días (Fowler \& Louzada 1996; Lescano et al. 2012). En consecuencia, las muestras de dietas fueron realizadas una semana anterior al muestreo de los basureros en cada uno de los nidos. De esta forma, podemos confiar con cierta certeza que la diversidad de compuestos orgánicos en los basureros provenía de la diversidad de la dieta estimada una semana antes.

Para determinar la relación de la actividad de los descomponedores (medida como su respiración) con la concentración de carbono y la diversidad de compuestos carbonados (estimada como la diversidad de la dieta), realizamos una regresión múltiple por pasos. Antes, realizamos una matriz de correlación entre las 3 variables independientes (concentración de carbono en los basureros, riqueza de especies en la dieta y diversidad de especies en la dieta) para detectar posibles correlaciones entre ellas. Como era de esperar, la riqueza y diversidad de especies vegetales en la dieta se encontraron altamente correlacionadas entre sí $(\mathrm{r}=0.83, P<0.01)$, pero no con la concentración de carbono (todos los $P>0.49$ ). En consecuencia, la diversidad de dieta fue eliminada del análisis. Para ajustarse a los supuestos, todas las variables del modelo fueron transformadas $\left(\log _{10}\right)$. El modelo final fue construido entonces con el $\log _{10}$ de la respiración como variable dependiente, y el $\log _{10}$ de la concentración de carbono en los basureros y el $\log _{10}$ de la riqueza de especies vegetales en la dieta como las variables independientes. Un nido que presentó datos atípicos en los análisis de laboratorio (nido 7) fue finalmente excluido del análisis estadístico, por lo cual nuestro número final de réplicas fue de 18 nidos. 


\section{Resultados}

Todas las variables estimadas mostraron gran variabilidad, permitiendo poner a prueba relaciones asociativas entre la actividad de microorganismos con la diversidad de dieta y concentración de carbono en los basureros. La actividad de los descomponedores estimada a partir de su respiración varió desde 1362 hasta $195 \mathrm{mg}$ de $\mathrm{CO}_{2} / 100 \mathrm{~g}$ de sustrato seco, dependiendo del nido muestreado. Por otra parte, encontramos gran variedad en la riqueza y la diversidad vegetal en la dieta de los nidos. El menor número de especies cosechadas por un hormiguero fue 2 y el mayor 14; el índice de diversidad de ShannonWiener varió entre 0.18 y 2.85 (Apéndice S2). Finalmente, la concentración de carbono en los basureros varió entre 90 y $435 \mathrm{~g} \mathrm{C} \mathrm{kg} \mathrm{por}$ kilo de sustrato seco.
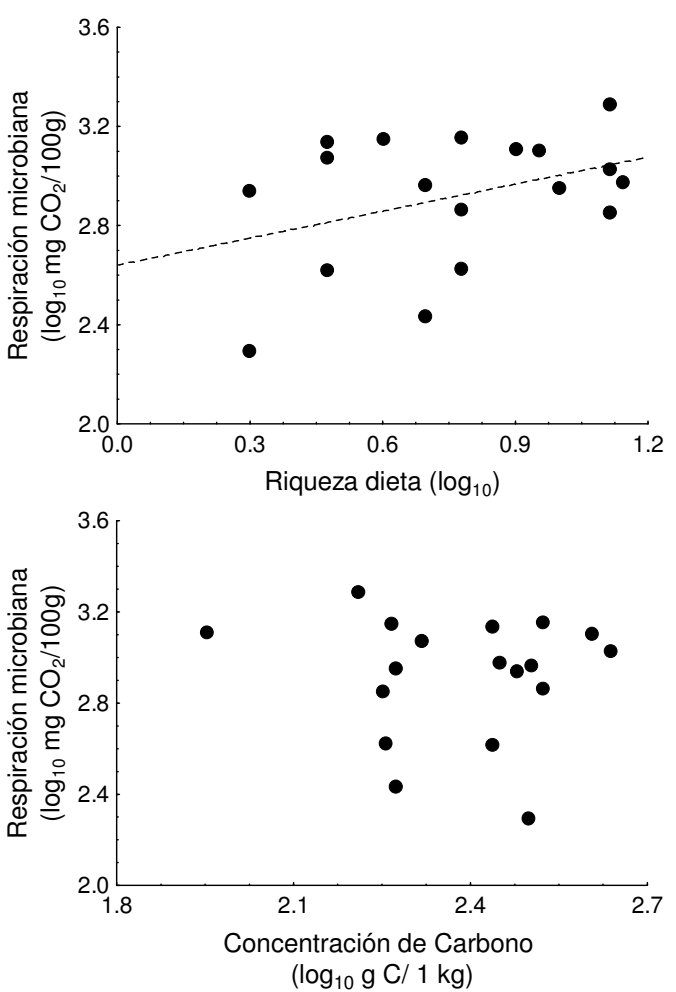

Figura 1. Relación de la respiración microbiana (medida como producción de $\mathrm{CO}_{2} / 100 \mathrm{~g}$ de sustrato seco durante un período de $72 \mathrm{~h}$, en condiciones controladas) con la riqueza de especies vegetales en la dieta (arriba) y la concentración de carbono en el basurero (abajo) de la hormiga cortadora de hojas Acromyrmex lobicornis. $\mathrm{N}=18$ hormigueros.

Figure 1. Relationship of the respiration rate of microorganisms (measured as production of $\mathrm{CO}_{2} / 100$ $\mathrm{g}$ of dry substrate during $72 \mathrm{~h}$, in lab conditions) with the plant species richness of their diet (above), and the concentration of carbonate compounds in external refuse dumps of the leaf-cutting ant Acromyrmex lobicornis (below). $\mathrm{N}=18$ nests.
La respiración de los microorganismos no se asoció con la concentración de carbono en los basureros, pero sí de forma parcial con la riqueza de la dieta de las hormigas. El modelo de regresión por pasos excluyó a la concentración de carbono en los basureros como posible variable explicativa $(t=0.07$, $P=0.95$ ), y retuvo parcialmente (i.e., aceptando un error de tipo $\mathrm{I}=0.11$ ) a la riqueza de especies $\left(F_{1,16}=2.71 ; P=0.11, R^{2}=0.15, b=0.38\right.$, Figura 1$)$.

\section{DISCUSIÓN}

Comprender que factores influyen sobre la actividad de los organismos descomponedores es especialmente relevante en sistemas áridos donde la abundancia y diversidad de materia orgánica es limitante (Naeem et al. 2000; Satti et al. 2003). Empleando los basureros de hormigas cortadoras de hojas, una de las fuentes de materia orgánica más importante en ambientes áridos, este trabajo sugiere que la actividad de los descomponedores no se incrementa con aumentos en la concentración de carbono de la materia orgánica a degradar, pero apoya de manera parcial la idea de que la actividad microbiana depende positivamente de la diversidad de compuestos carbonados disponibles para descomponer.

La variación en la concentración de carbono en los basureros no se asoció con la respiración de los microorganismos. Eso puede deberse a que no todos los compuestos carbonados se degradan de igual manera. Algunas especies de plantas son ricas en compuestos carbonados fácilmente degradables, mientras que otras poseen gran concentración de compuestos resistentes a la degradación, como lignina (Davidson \& Janssens 2006; Gessner et al. 2010). Muchas de las especies consumidas por A. lobicornis son pastos, hierbas o plantas con hojas duras o espinosas, resistentes a la desecación y al ataque de grandes herbívoros (Apéndice S2). En consecuencia, es posible que buena parte de la concentración de carbono obtenida en el laboratorio provenga de compuestos carbonados cuya descomposición no sea sencilla para los microorganismos. Por lo cual, al menos en este caso, la concentración de carbono "per se" no afectaría la tasa de actividad de los descomponedores.

Por otra parte, nuestros resultados sugieren que la diversidad de materia orgánica favorece la actividad de los descomponedores, ya que la respiración en los basureros mostró una tendencia a asociarse de forma positiva con la riqueza de especies vegetales en la dieta, 
una aproximación a la diversidad de materia orgánica disponible para degradar. Dado que la actividad de los microorganismos está positivamente relacionada con la productividad de los ecosistemas (Raich \& Tufekcioglu 2000; Rustad et al. 2001; Jassal et al. 2007; Zak et al. 2003; Thomey et al. 2011; Caprez et al. 2012 y referencias allí citadas), este resultado podría aportar evidencias indirectas para comprender mejor la relación entre diversidady productividad. Tradicionalmente, los ecólogos han enfocado sus esfuerzos en comprender cómo ciertas características del ecosistema regulan la biodiversidad; en otras palabras, la biodiversidad ha sido considerada como la variable "dependiente". En general, estos trabajos han encontrado una relación positiva cuya forma depende del grupo taxonómico estudiado, escala geográfica del estudio y tipo de enfoque, entre otros factores (Abrams 1995). Sin embargo, en las últimas décadas varios estudios han considerado que la productividad puede depender de la diversidad, considerando a la productividad como la variable dependiente (Cardinale et al. 2009). La tendencia encontrada de una mayor actividad microbiana ante incrementos en la diversidad de recursos para degradar podrían considerarse un resultado consistente con la hipótesis de facilitación-complementariedad, que predice una relación positiva entre la diversidad y la productividad (Naeem et al. 2000; Loreau 2001; Gessner et al. 2010). Sin embargo, esta interpretación debe considerarse con cuidado, dado que nuestro enfoque presenta ciertas debilidades. Primero, la diversidad de recursos para los descomponedores es estimada indirectamente a través de la diversidad de la dieta de las hormigas, lo cual puede ser sesgado. Como fuera explicado antes, los diferentes compuestos carbonados que forman los tejidos vegetales se encuentran en distinta proporción según la especie de planta y los descomponedores no son capaces de aprovechar todos los compuestos de la misma forma (Davidson \& Janssens 2006; Abril 2011; Scott et al. 2010). Este proceso hace que la diversidad de compuestos orgánicos en los basureros pueda no ser necesariamente similar a la diversidad de plantas cosechadas por las hormigas. Segundo, por cuestiones metodológicas y logísticas, en este trabajo no estimamos la diversidad de descomponedores en los basureros, información clave para poner a prueba la hipótesis de facilitacióncomplementariedad. Sería conveniente estimar la diversidad de compuestos carbonados además de la diversidad de plantas cosechadas, y determinar la identidad de los organismos descomponedores en los basureros para explicar mejor la relación entre actividad microbiana y diversidad en los basureros (ver Scott et al. 2010). Tercero, debería probarse si una mayor tasa de respiración microbiana se asocia con una mayor productividad, relación no verificada en este estudio. Sin embargo, el sistema en estudio presenta ciertas virtudes. Primero, los basureros de las hormigas pueden ser buenos modelos para poner a prueba si la productividad depende de la diversidad sin confusiones relacionadas con efectos recíprocos de causa-efecto, ya que teóricamente es factible que la actividad de los descomponedores pueda depender de la diversidad de materia orgánica en los basureros, pero no viceversa (o sea, es poco lógico suponer que la diversidad de materia orgánica proveniente de la diversidad de dieta de las hormigas dependa de la actividad de los descomponedores). Segundo, nuestro enfoque correlativo devela la existencia de una gran variación en los niveles de respiración de microorganismos descomponedores en los basureros de las hormigas. Esta variabilidad hace de los basureros de hormigas cortadoras un sistema ideal para estudiar con más profundidad los factores del sustrato que influyen sobre la actividad microbiana. En ese sentido, este trabajo es pionero. Finalmente, el sistema aquí estudiado ilustra la importancia de los efectos indirectos, revelando cómo un empobrecimiento en los ensambles de plantas puede reducir la actividad de los microorganismos descomponedores a través de cambios en la dieta de las hormigas.

La contribución de materia orgánica de las hormigas cortadoras de hojas es de vital importancia en sitios donde tradicionalmente la abundancia y la diversidad de materia orgánica es limitante, como en los sistemas desérticos (Bestelmeyer \& Wiens 2003; FarjiBrener 2010). Los basureros de las hormigas cortadoras son varias veces más ricos en carbono orgánico y poseen mayor capacidad de retención de humedad que los suelos adyacentes (Farji-Brener \& Ghermandi 2000; 2004; Abril 2011). En consecuencia, ofrecen condiciones ideales para la reproducción y abundancia de la biota del suelo (FarjiBrener 2010; Sousa-Souto et al. 2011). Esta gran actividad de los descomponedores genera una alta concentración de nutrientes en los basureros (Farji-Brener \& Tadey 2009) favoreciendo el crecimiento y desarrollo de la plantas alrededor de los nidos (FarjiBrener \& Ghermandi 2008; Farji-Brener et al. 
2010; Cerdá et al. 2012). Si las tendencias que encontramos en este trabajo se confirman, la abundancia de nutrientes disponibles para las plantas en los basureros debido a la actividad de los descomponedores va a depender, en última instancia, de la diversidad de la dieta de las hormigas. Basureros de nidos en ambientes poco disturbados, con una buena diversidad de vegetación, van presentar una tasa de descomposición más alta que basureros de nidos en sitios con poca diversidad de vegetación, generando un efecto positivo de "cascada hacia arriba" sobre otros niveles tróficos (ver Lescano et al. 2012). Apoyando esta idea, un trabajo reciente ha demostrado como el ganado, al empobrecer la vegetación y reducir entonces la diversidad de dieta de las hormigas, disminuye de manera indirecta la calidad nutritiva de sus basureros (Tadey \& Farji-Brener 2007). Este estudio no sólo ilustra cómo estas hormigas afectan indirectamente los suelos en ambientes áridos, sino que brinda otro novedoso ejemplo de cómo el empobrecimiento de la vegetación puede afectar de forma negativa la actividad de los descomponedores de los ecosistemas vía organismos intermediarios.

Agradecimientos: Este proyecto fue financiado parcialmente por la Agencia Nacional de Promoción Científica y Técnica (FONCyT) mediante el PICTOUNRN 2010-0179 adjudicado a AGFB. Tres revisores anónimos realizaron comentarios que mejoraron una versión preliminar de este manuscrito.

\section{BIBLIOGRAFÍA}

AbrAms, PA. 1995. Monotonie or unimodal diversity productivity gradients: what does competition theory predict? Ecology, 76:2019-2027.

ABriL, A. 2011. The Leaf-Cutting Ant-Plant Interaction from a Microbial Ecology Perspective. Part 2. Pp. 3763 en: Seckbach, J \& Z Dubinsky (eds.). All Flesh Is Grass. Cellular Origin, Life in Extreme Habitats and Astrobiology Book Series 16, Springer-Verlag, New York, EEUU.

ABrIL, AB \& EH BUCHER. 2002. Evidence that the fungus cultured by leaf-cutting ants does not metabolize cellulose. Ecol. Lett., 5:325-328.

ABRIL, AB \& EH BUCHER. 2004. Nutritional sources of the fungus by leaf-cutting ants. Appl. Soil Ecol., 26:243-247.

Aguiar, M \& O Sala. 1994. Competition, facilitation, seed distribution and the origin of patches in a Patagonian steppe. Oikos, 70:26-34.

Anderson, JP. 1982. Soil respiration. Pp. 831-871 en: Page, AL \& RH Miller (eds.). Methods of Soil Analysis. Part 2. Chemical and Microbiological Properties. Agronomy Monograph. 9, $2^{\text {nd }}$ Ed. ASA, CSSA, SSSA, Madison, WI, EEUU.

Bestelmeyer, BT \& J Wiens. 2003. Scavenging ant foraging behavior and variation in the scale of nutrient redistribution among semi-arid grasslands. J. Arid Environ., 53:373-386.
BuRKART, R; NO BÁRBARO; RO SÁNCHEZ \& DA GÓMEZ. 1999. Eco-regiones de la Argentina. Componente Educación Ambiental del PRODIA, APN, Buenos Aires, Argentina. Pp. 43.

CABrerA, AL. Regiones Fitogeográficas Argentinas. 1976. En: Enciclopedia Argentina de Agricultura y Jardinería. Tomo 2. Ed. Acme SA, Buenos Aires, Argentina. Pp. 85.

Caprez, R; Niklaus, P; \& C Körner. 2012. Forest soil respiration reflects plant productivity across a temperature gradient in the Alps. Oecologia, 170: 1143-1154

Cardinale, B; B Bennett; C Nelson \& K Gross. 2009. Does productivity drive diversity or vice versa? A test of the multivariate productivity-diversity hypothesis in streams. Ecology, 90:1227-1241.

Cerda, NV; M TAdeY; AG FarJi-Brener \& MC NAVARro. 2012. Effects of leaf-cutting ant refuse on native plant performance under two level of grazing intensity in the Monte Desert of Argentina. Appl. Veg. Sci., 15:479-487.

COOPERBAND, LR \& JH MidDETON. 1996. Changes in chemical, physical and biological properties of passively-aerated co composted poultry litter and municipal solid waste compost. Compost Sci. Util., 4:24-34.

DAVIDSON, EA \& IA JANSSENS. 2006. Temperature sensitivity of soil carbon decomposition and feedbacks to climate change. Nature, 440:165-173

Del VALLE, H. 1998. Patagonian soils: a regional synthesis. Ecología Austral, 8:103-123.

FARJI-BRENER, AG. 2000. Leaf-cutting ant nests in temperate environments: mounds, mound damages and mortality rates in Acromyrmex lobicornis. Stud. Neotrop. Faun. $\mathcal{E}$ Environm., 35:131-138.

FARJI-BREner, AG. 2010. Leaf-Cutting Ant Nests and Soil Biota Abundance in a Semi-Arid Steppe of Northwestern Patagonia. Sociobiology, 56:549-557

FARJI-Brener, AG \& L GHERMANDI. 2000. The influence of nests of leaf-cutting ants on plant species diversity in road verges of northern Patagonia. J. Veg. Sci., 11: 453-460.

FArJi-Brener, AG \& L Ghermandi. 2004. Seedling recruitment in a semi-arid Patagonian steppe: Facilitative effects of refuse dumps of leaf-cutting ants. J. Veg. Sci., 15:823-830.

FARJI-BREner, AG \& L GHERMANDI. 2008. Leaf-cutting ant nests near roads increase fitness of exotic plant species in natural protected areas. P. Roy. Soc. Lond. B, 275: 1431-1440.

FARJ-Brener, AG \& A Ruggiero. 1994. Leaf-cutting ants (Atta and Acromyrmex) inhabiting Argentina: patterns in species richness and geographical range sizes. J. Biogeogr., 21:391-399.

FARJI-Brener, AG \& C Medina. 2000. The importance of where to dump the refuse: seed banks and fine roots in nests of the leaf-cutting ants Atta cephalotes and Atta colombica. Biotropica, 32:120-126.

FARJI-BRENER, AG \& A ILLES. 2000. Do leaf-cutting ant nests make 'bottom up' gaps in neotropical rain forests? A critical review of the evidence. Ecol. Lett., 3:219-227.

FARJI-BRENER, AG \& M TADEY. 2009. Contributions of leafcutting ants to soil fertility: causes and consequences. Pp. 81-91 en: Lucero, DP \& JE Boggs. Soil fertility. Nova Science Publishers Inc., Nueva York, EEUU.

Farji-Brener, AG; N Lescano \& L Ghermandi. 2010. Ecological engineering by a native leaf-cutting ant increases the performance of exotic plant species. Oecología, 163:163-169. 
Fowler, HG \& J LouzadA. 1996. Refuse production in leaf-cutting ants (Hymenoptera: Formicidae) and its relationship with leaf harvest. Biol. Rhythm. Res., 27: 148-152.

Franzel, C \& AG Farji-Brener. 2000. ¿Oportunistas o selectivas? Plasticidad en la dieta de la hormiga cortadora de hojas Acromyrmex lobicornis en el noroeste de la Patagonia. Ecología Austral, 10:159-168.

Gessner, MO; CM Swan; CK Dang; BG McKie; RD BARDGETT; ET AL. 2010. Diversity meets decomposition. TREE, 25:372-380.

HalouA-Gruneisen, P. 1996. La Vegetación de Monte en el Yacimiento Aguada de la Pichana. Argentina. Total Austral, Buenos Aires, Argentina. Pp. 43.

Iannotti, DA; ME Grebus; BL Coth; LV Madden \& H HoITINK. 1994. Oxygen respirometry to assess stability and maturity of composted municipal solid waste. J. Environ. Qual., 23:1177-1183.

Jassal, RS; TA Black; T CaI; K Morgenstern; Z LI; et al. 2007. Components of ecosystem respiration and an estimate of net primary productivity of an intermediateaged Douglas-fir stand. Agric. Forest Meteor., 144:44-57

KANDELER, E. 2007. Physiological and biochemical methods for studying soil biota and their function. Cap. 3. Pp. 5384 en: Paul, EA (ed.). Soil microbiology and biochemistry. $3^{\text {ra }}$ Ed. Academic Press, New York, EEUU.

Leconte, MC; MJ Mazzarino; P SATti; MC Iglesias \& F LAOS. 2009. Co-composting rice hulls and/or sawdust with poultry manure in NE Argentina. Waste Manage., 29:2446-2453

Lescano N; AG FarjI-Brener; E Gianoli \& T Carlo. 2012. Bottom-up effects may not reach the top: the influence of ant-aphid interactions on the spread of soil disturbances through trophic chains. P. Roy. Soc. Lond. B., 279:37793787.

Loreau, M. 2001. Microbial diversity, producerdecomposer interactions and ecosystem processes: a theoretical model. P. Roy. Soc. Lond. B Bio., 268:303309.

Mermoz, M \& C MARTIN. 1986. Mapa de vegetación del Parque y Reserva Nacional Nahuel Huapi. Administración de Parques Nacionales, Delegación Técnica Regional Patagonia. Informe Técnico. Pp. 23.

Moller, I.; H Licht; J Harholt; W Willats \& J BoOmSma. 2011. The Dynamics of Plant Cell-Wall Polysaccharide Decomposition in Leaf-Cutting Ant Fungus Gardens. PlosOne, 6(3) e17506.

Naeem, S; DR Hahn \& G SchuUrman. 2000. Producerdecomposer co-dependency influences biodiversity effects. Nature, 403:762-764.
Nelson, DW \& LE Sommers. 1996. Total Carbon, Organic Carbon, and Organic Matter. Pp. 961-1010 en: Sparks, DL; Al Page; PA Helmke \& RH Loeppert. Methods of Soil Analysis, Part 3. Madison, WI, EEUU.

ORCHARD, VA \& FJ COOK. 1983. Relationship between soil respiration and soil moisture. Soil Biol. Biochem., 15:447-453.

Pilati, A; EM Quirán, \& HD Estelrich. 1997. Actividad forrajera de Acromyrmex lobicornis Emery (Hymenoptera: Formicidae) en un pastizal natural semiárido en la provincia de La Pampa (Argentina). Ecología Austral, 7:49-56.

Raich, J \& A Tufekcioglu. 2000. Vegetation and soil respiration: correlations and controls. Biogeochemistry, 48:71-90.

Rustad, L; JL Campbell; G Marion; RJ Norby; MJ Mitchell; ET AL. 2001. A meta-analysis of the response of soil respiration, net nitrogen mineralization, and aboveground plant growth to experimental ecosystem warming. Oecologia, 126:543-562

SAtTI, P; MJ Mazzarino; M GobBi; F Funes; L Roselli: et AL. 2003. Soil N dynamics in relation to leaf litter quality and soil fertility in north-western Patagonian forests. J. Ecol., 91:173-181.

Scott, JJ; KJ BudsberG; G Suen; DL Wixon; TC Balser; et AL. 2010. Microbial community structure of leaf-cutter ant fungus gardens and refuse dumps. PLoS One, 5, e9922.

Sousa-Souto, L; D Jesús Santos; B Ambrogi; M Campos dos SANTOS; M BRAGA BuENO GUERRAD; ET AL. 2011. Increased $\mathrm{CO}_{2}$ emission and organic matter decomposition by leaf-cutting ant nests in a coastal environment. Soil Biol. Biochem., 30:1-5.

TADEY, M \& AG FARJI-BRENER. 2007. Indirect effects of exotic grazers: livestock decreases the nutrient content of refuse dumps of leaf-cutting ants through vegetation impoverishment. J. Appl. Ecol., 44:1209-1218.

THOMEY, M; S COLlins; R VARGAS; J JHONSON; R BROWN; ET AL. 2011. Effect of precipitation variability on net primary production and soil respiration in a Chihuahuan Desert grassland. Global Change Biology, 17:1505-1515

TognetTI, C; MJ Mazzarino \& F LAOS. 2007. Improving the quality of municipal organic waste compost. Bioresource Technol., 98:1067-1076.

Wirth, R; H HeRz; RJ RYel; W Beyschlag \& B HÖLldobler. 2003. Herbivory of leaf-cutting ants. Springer, Berlin. Pp. 228.

ZaK, DR; W Holmes; D White; A Peacock \& D Tilman. 2003. Plant diversity, soil microbial communities, and ecosystem function: are there any links? Ecology, 84: 2042-2050. 


\section{INFORMACIÓN SUPLEMENTARIA}

Apéndice S1. Ubicación y características generales de los sitios de muestreo.

${ }^{*}$ ) Nido excluido del análisis por datos anormales en los análisis de laboratorio (nido 7).

Appendix S1. Location and characteristics of sample sites.

(*) Nest excluded from the analyses because of atypical values in the lab analyses (nest 7).

\begin{tabular}{|c|c|c|c|c|}
\hline Localidad & Sitio & Nido $n^{\circ}$ & Características & Usos \\
\hline Neuquén & Barda & 1,2 y 3 & $\begin{array}{l}\text { Zona urbana de la ciudad, se destaca } \\
\text { por su geología caracterizada por } \\
\text { terrazas y depósitos de antiguas } \\
\text { llanuras aluviales }\end{array}$ & Actividades recreativas \\
\hline Neuquén & Plantación de Pinos & 4 & $\begin{array}{l}\text { Riqueza de especies vegetales baja, } \\
\text { afectada por plantación de pinos. } \\
\text { Región de bardas, zona urbana }\end{array}$ & Actividades recreativas \\
\hline Neuquén & $\begin{array}{l}\text { Ex plantación de } \\
\text { manzanas }\end{array}$ & $5,6,7^{*}$ y 8 & $\begin{array}{l}\text { Antiguamente utilizado para el } \\
\text { cultivo de manzanas, abandonado } \\
\text { hace aproximadamente } 5 \text { años }\end{array}$ & \\
\hline Mari Menuco & Mari Menuco & 9,10 y 11 & A orillas del lago Mari Menuco & $\begin{array}{l}\text { Esporádicamente utilizado } \\
\text { para realizar caminatas }\end{array}$ \\
\hline Neuquén & Plaza & 12 & $\begin{array}{l}\text { Plaza ubicada en la zona céntrica de } \\
\text { la ciudad }\end{array}$ & Actividades recreativas \\
\hline Villa Llanquin & Ruta & 13 y 14 & $\begin{array}{l}\text { Borde de ruta } 40 \text {, a } 100 \mathrm{~m} \text { del río } \\
\text { Limay }\end{array}$ & $\begin{array}{l}\text { Parque Nacional Nahuel } \\
\text { Huapi }\end{array}$ \\
\hline Villa Llanquin & Sector escalada & 15,16 y 17 & Cercano a la zona urbana & Pastoreo y en verano zona de \\
\hline Dina Huapi & Dina Huapi & 18 y 19 & $\begin{array}{l}\text { Borde de ruta, cercano a zona urbana, } \\
500 \text { m de la confluencia del lago } \\
\text { Nahuel Huapi y el río Limay }\end{array}$ & \\
\hline
\end{tabular}

Apéndice S2. Lista de especies vegetales cosechadas. El nido 7 fue excluido del análisis por presentar valores atípicos en los análisis de laboratorio. Entre paréntesis se detalla la frecuencia relativa (en \%) de cada especie para cada colonia.

Appendix S2. List of the plant species harvested. The nest 7 was excluded from the analysis because of its atypical values in the lab analyses. The relative frequency (in \%) of each plant species is given in parenthesis

\begin{tabular}{|c|c|c|c|c|}
\hline Nido & Especies forrajeadas & $\begin{array}{c}\text { Frecuencia absoluta y } \\
\text { relativa }(\%)\end{array}$ & Riqueza & $\begin{array}{c}\text { Shannon- } \\
\text { Wiener }\end{array}$ \\
\hline \multirow{14}{*}{1} & Sphaeralcea mendocina & $12(3.0)$ & 13 & 2.27 \\
\hline & Atriplex lampa & $11(2.7)$ & & \\
\hline & Chuquiraga erinacea & $4(1.0)$ & & \\
\hline & Maihueniopsis darwinii & $2(0.5)$ & & \\
\hline & Senecio pinnatus & $9(2.2)$ & & \\
\hline & Sp 4 & $5(1.2)$ & & \\
\hline & Bougainvillea spinosa & $98(24.4)$ & & \\
\hline & Gutierrezia solbrigii & $126(31.3)$ & & \\
\hline & Cercidium praecox & $3(0.7)$ & & \\
\hline & Plantago patagonica & $1(0.2)$ & & \\
\hline & Prosopis alpataco & $1(0.2)$ & & \\
\hline & Cyclolepis genistoides & $2(0.5)$ & & \\
\hline & Larrea divaricata & $25(6.2)$ & & \\
\hline & Palitos leñosos & $103(25.6)$ & & \\
\hline \multirow[t]{4}{*}{2} & Senecio pinnatus & $84(23.2)$ & 3 & 0.30 \\
\hline & Bougainvillea spinosa & $3(0.8)$ & & \\
\hline & Atriplex lampa & $1(0.3)$ & & \\
\hline & Palitos leñosos & $274(75.7)$ & & \\
\hline \multirow[t]{9}{*}{3} & Gutierrezia solbrigii & $53(24.3)$ & 8 & 1.67 \\
\hline & Sphaeralocea mendocina & $25(11.5)$ & & \\
\hline & Larrea cuneifolia & $2(0.9)$ & & \\
\hline & Senecio pinnatus & $2(0.9)$ & & \\
\hline & Bougainvillea spinosa & $1(0.5)$ & & \\
\hline & Maihueniopsis darwinii & $1(0.5)$ & & \\
\hline & Atriplex lampa & $2(0.9)$ & & \\
\hline & Grindelia chiloensis & $4(1.8)$ & & \\
\hline & Palitos leñosos & $128(58.7)$ & & \\
\hline \multirow[t]{2}{*}{4} & Cyclolepis genistoides & $150(73.5)$ & 6 & 1.11 \\
\hline & Sphaeralcea mendocina & $6(2.9)$ & & \\
\hline
\end{tabular}


Apéndice S2. Continuación.

Appendix S2. Continuation.

\begin{tabular}{|c|c|c|c|c|}
\hline Nido & Especies forrajeadas & $\begin{array}{c}\text { Frecuencia absoluta y } \\
\text { relativa }(\%)\end{array}$ & Riqueza & $\begin{array}{l}\text { Shannon- } \\
\text { Wiener }\end{array}$ \\
\hline & Senecio pinnatus & $9(4.4)$ & & \\
\hline & Plantago patagonica & $6(2.9)$ & & \\
\hline & Larrea cuneifolia & $9(4.4)$ & & \\
\hline & Pinus $s p$ & $4(2.0)$ & & \\
\hline & Palitos leñosos & $20(9.8)$ & & \\
\hline \multirow[t]{4}{*}{5} & Sp 1 & $97(31.3)$ & 3 & 1.24 \\
\hline & Gramínea & $21(6.8)$ & & \\
\hline & Populus alba & $27(8.7)$ & & \\
\hline & Palitos leñosos & $165(53.2)$ & & \\
\hline \multirow[t]{3}{*}{6} & Populus alba & $11(3.8)$ & 2 & 0.34 \\
\hline & Sp 2 & $161(55.1)$ & & \\
\hline & Palitos leñosos & $120(41.1)$ & & \\
\hline \multirow[t]{5}{*}{7} & Sp 3 & 177 (49.7) & 4 & 1.25 \\
\hline & Sp 2 & $68(19.1)$ & & \\
\hline & Populus alba & $25(7.0)$ & & \\
\hline & Gramíneas & $1(0.3)$ & & \\
\hline & Palitos leñosos & $85(23.9)$ & & \\
\hline \multirow[t]{4}{*}{8} & Populus alba & $116(35.4)$ & 3 & 1.09 \\
\hline & Sp 2 & $104(31.7)$ & & \\
\hline & Gramíneas & $3(0.9)$ & & \\
\hline & Palitos leñosos & $105(32.0)$ & & \\
\hline \multirow[t]{11}{*}{9} & Sp 32 & $91(14.7)$ & 10 & 2.64 \\
\hline & Prosopis alpataco & $9(1.5)$ & & \\
\hline & Bougainvillea spinosa & $59(9.5)$ & & \\
\hline & Gutierrezia solbrigii & $43(6.9)$ & & \\
\hline & Gramínea & $37(6.0)$ & & \\
\hline & Fabiana sp. & $39(6.3)$ & & \\
\hline & Larrea divaricata & $10(1.6)$ & & \\
\hline & Phacelia artemisioides & $4(0.6)$ & & \\
\hline & Atriplex lampa & $1(0.2)$ & & \\
\hline & Sp 33 & $3(0.5)$ & & \\
\hline & Palitos leñosos & $324(52.3)$ & & \\
\hline \multirow[t]{6}{*}{10} & Fabiana sp. & $45(9.7)$ & 5 & 1.48 \\
\hline & Gutierrezia solbrigii & $16(3.5)$ & & \\
\hline & Sp 32 & $9(1.9)$ & & \\
\hline & Bougainvillea spinosa & $171(36.9)$ & & \\
\hline & Larrea sp. & $14(3.0)$ & & \\
\hline & Palitos leñosos & $208(44.9)$ & & \\
\hline \multirow[t]{5}{*}{11} & Larrea divaricata & $39(10.3)$ & 4 & 1.61 \\
\hline & Fabiana sp. & $53(14.0)$ & & \\
\hline & Atriplex lampa & $2(0.5)$ & & \\
\hline & Bougainvillea spinosa & $23(6.1)$ & & \\
\hline & Palitos Leñosos & $262(69.1)$ & & \\
\hline \multirow[t]{6}{*}{12} & Trifolium sp. & $65(46.1)$ & 5 & 1.39 \\
\hline & Populus alba & $15(10.6)$ & & \\
\hline & Plantago lanceolata & $2(1.4)$ & & \\
\hline & Sp 30 & $6(4.3)$ & & \\
\hline & Sp 31 & $5(3.5)$ & & \\
\hline & Palitos leñosos & $48(34.0)$ & & \\
\hline \multirow[t]{10}{*}{13} & Sp 15 & $28(7.2)$ & 9 & 1.93 \\
\hline & Fabiana imbricata & $84(21.5)$ & & \\
\hline & Sp 16 & $17(4.4)$ & & \\
\hline & Gramínea & $6(1.5)$ & & \\
\hline & Cirsium sp. & $19(4.9)$ & & \\
\hline & Centaurea solstitialis & $179(45.9)$ & & \\
\hline & Dysphania multifida & $2(0.5)$ & & \\
\hline & Sp 17 & $1(0.3)$ & & \\
\hline & Sp 7 & $1(0.3)$ & & \\
\hline & Palitos leñosos & $53(13.6)$ & & \\
\hline
\end{tabular}


Apéndice S2. Continuación.

Appendix S2. Continuation.

\begin{tabular}{|c|c|c|c|c|}
\hline Nido & Especies forrajeadas & $\begin{array}{c}\text { Frecuencia absoluta y } \\
\text { relativa }(\%)\end{array}$ & Riqueza & $\begin{array}{l}\text { Shannon- } \\
\text { Wiener }\end{array}$ \\
\hline \multirow[t]{15}{*}{14} & Sp 15 & $65(17.6)$ & 14 & 2.85 \\
\hline & Baccharis sp. & $48(13.0)$ & & \\
\hline & Cirsium sp. & $71(19.2)$ & & \\
\hline & Sp 16 & $7(1.9)$ & & \\
\hline & Centaurea solstitialis & $17(4.6)$ & & \\
\hline & Gramínea & $9(2.4)$ & & \\
\hline & Fabiana imbricata & $6(1.6)$ & & \\
\hline & Sp 7 & $1(0.3)$ & & \\
\hline & Sp 18 & $34(9.2)$ & & \\
\hline & Sp 19 & $2(0.5)$ & & \\
\hline & Sp 20 & $9(2.4)$ & & \\
\hline & Sp 21 & $1(0.3)$ & & \\
\hline & Sp 22 & $2(0.5)$ & & \\
\hline & Sp 23 & $2(0.5)$ & & \\
\hline & Palitos leñosos & $96(25.9)$ & & \\
\hline \multirow[t]{3}{*}{15} & Gramíneas & $9(2.7)$ & 2 & 0.18 \\
\hline & Populus alba & $325(95.9)$ & & \\
\hline & Palitos leñosos & $5(1.5)$ & & \\
\hline \multirow[t]{14}{*}{16} & Sp 5 & $7(1.5)$ & 13 & 2.33 \\
\hline & Sp 6 & $10(2.1)$ & & \\
\hline & Trifolium sp. & $30(6.4)$ & & \\
\hline & Gramínea & $3(0.6)$ & & \\
\hline & Sp 7 & $7(1.5)$ & & \\
\hline & Junellia sp. & $17(3.6)$ & & \\
\hline & Sp 8 & $5(1.1)$ & & \\
\hline & Sp 9 & $3(0.6)$ & & \\
\hline & Populus alba & $16(3.4)$ & & \\
\hline & Baccharis sp. & $153(32.8)$ & & \\
\hline & Rosa rubiginosa & $182(39.0)$ & & \\
\hline & Sp 10 & $8(1.7)$ & & \\
\hline & Sp 11 & $5(1.1)$ & & \\
\hline & Palitos leñosos & $21(4.5)$ & & \\
\hline \multirow[t]{14}{*}{17} & Baccharis sp. & $124(32.6)$ & 13 & 2.37 \\
\hline & Sp 12 & $4(1.1)$ & & \\
\hline & Trifolium sp. & $90(23.7)$ & & \\
\hline & Populus alba & $4(1.1)$ & & \\
\hline & Senecio bracteolatus & $1(0.3)$ & & \\
\hline & Sp 8 & $16(4.2)$ & & \\
\hline & Rosa rubiginosa & $12(3.2)$ & & \\
\hline & Gramínea & $2(0.5)$ & & \\
\hline & Sp 11 & $15(3.9)$ & & \\
\hline & Abrojo & $1(0.3)$ & & \\
\hline & Sp 10 & $14(3.7)$ & & \\
\hline & Sp 14 & $2(0.5)$ & & \\
\hline & Sp 13 & $9(2.4)$ & & \\
\hline & Palitos leñosos & $86(22.6)$ & & \\
\hline \multirow[t]{7}{*}{18} & Plantago lanceolata & $14(5.6)$ & 6 & 1.89 \\
\hline & Rumex acetosella & $58(23.2)$ & & \\
\hline & Acaena caespitosa & $8(3.2)$ & & \\
\hline & Populus alba & $4(1.6)$ & & \\
\hline & Sp 28 & $22(8.8)$ & & \\
\hline & Sp 29 & $2(0.8)$ & & \\
\hline & Palitos leñosos & $142(56.8)$ & & \\
\hline \multirow[t]{7}{*}{19} & Sp 24 & $12(15.6)$ & 6 & 2.37 \\
\hline & Sp 25 & $4(5.2)$ & & \\
\hline & Sp 26 & $4(5.2)$ & & \\
\hline & Sp 27 & $9(11.7)$ & & \\
\hline & Rumex acetosella & $67.8)$ & & \\
\hline & Gramíneas & $2(2.6)$ & & \\
\hline & Palitos leñosos & $40(51.9)$ & & \\
\hline
\end{tabular}

